\title{
Long-term follow-up of cystic thyroid nodules treated with percutaneous ethanol injection (PEI) using two different approaches
}

\author{
M Deandrea ${ }^{1}$, P Trimboli², A Creanza', F Garino ${ }^{1}$, A Mormile', S Bertolino³, R Garberoglio', P P Limone ${ }^{1}$ and \\ M Zingrillo ${ }^{4}$ \\ ${ }^{1}$ Endocrinology, Diabetes and Metabolism Department and Center for Thyroid Diseases, A.O. Ordine Mauriziano, \\ Turin, Italy, ${ }^{2}$ Clinic for Nuclear Medicine and Competence Center for Thyroid Diseases, Imaging Institute of Southern \\ Switzerland, Ente Ospedaliero Cantonale, Bellinzona, Switzerland, ${ }^{3}$ Medicine Department, University of Foggia, \\ Foggia, Italy, and ${ }^{4}$ Thyroid Private Outpatient Office, Foggia, Italy \\ Correspondence \\ should be addressed \\ to M Deandrea \\ Email \\ mdeandrea@mauriziano.it
}

\begin{abstract}
Background and aim: Cystic thyroid nodules (CNs), although generally benign, can cause compressive or aesthetic problems. Percutaneous ethanol injection (PEI) can represent an alternative to surgery. The present retrospective study evaluates: (i) the long-term outcome of CNs after PEl; (ii) the differences between two different PEI protocols; (iii) the $\mathrm{CNs}$ response according to the liquid component.

Materials and methods: The study comprises 358 nodules post-PEI followed for at least 2 years. PEl was performed according to two different treatment protocols with a single (Foggia) or double (Turin) alcohol injection. CNs were divided according to their composition: cystic (CYS) >90\%, mainly cystic (M-CYS) 75-90\%, mixed (MIX) 50-75\%, solidmixed (S-MIX) 35-50\%. The volume reduction rate (VRR) was defined as nodule volume $(\mathrm{mL})$ after PEI/nodule volume $(\mathrm{mL})$ before PEI x 100.

Results: The 1-year VRR was significantly higher than that at 6 months ( $89.5 \%$ vs $72.9 \%, P=0.0005)$, no differences were observed after 1 year. A significant difference between Turin and Foggia was observed only in VRR at early visit (79\% vs $86 \%$, respectively, $P=0.002)$ and recurrence rate (14\% vs $24 \%$, respectively, $P=0.001)$. Minor side-effects were infrequent. In 192 nodules with a 10-year follow-up CYS showed higher VRR than MIX and S-MIX nodules $(P<0.001)$. Conclusion: Our study reported that the long-term outcome of CNs treated with PEI is excellent regardless of the PEI technique utilized; the larger the cystic amount, the higher the VRR. Based on present results, PEI can be considered as the first-line choice for treating thyroid CNs.
\end{abstract}

\section{Introduction}

Cystic thyroid nodules (CNs) are defined as enlarged fluid-filled lesions of the thyroid. They may be entirely cystic or complex cysts with some solid parts. Because $\mathrm{CNs}$ are generally benign with very rare exception, the current guidelines indicate that diagnostic aspirate is not necessary (1). On the other hand, a $\mathrm{CN}$ can result in compressive symptoms or aesthetic concerns depending on its size and location. In this case, surgery can be (c) 2020 European Society of Endocrinology Printed in Great Britain
European Journal of Endocrinology

(2020) 183, 489-495 considered as excessive and alternatives are strongly recommended. Percutaneous draining of fluids represents the initial non-surgical option which also allows the cytological assessment. However, after fluid removal, the majority of CNs recur (1). The percutaneous ethanol injection (PEI) represents the second-line treatment. PEI has been shown to be a safe and effective alternative to the surgical excision $(2,3,4,5,6,7)$ in order to achieve 
significant improvement of symptoms and health-related quality of life $(8,9,10)$. Nevertheless, regardless of an extensive published information on the high reliability of PEI in both reducing the size of CNs and controlling the related symptoms, this therapeutic option has not been fully accepted by the international thyroid community and is not always included in clinical guidelines $(11,12)$. One main reason of the latter could be the very poor information on the long-term outcome of CNs after PEI treatment. Furthermore, a standardized PEI procedure has not been yet established, and there are significant differences among authors regarding important issues, such as volume of alcohol to instill, re-aspiration or retention of alcohol, number and interval of PEI sessions, and length of follow-up to plan $(13,14,15,16)$. Analyzing data on long-term post-PEI follow-up of CNs treated with different approaches could significantly contribute to achieve a solid information on its reliability and therefore promoting the use thereof.

According to the aforementioned issues, the aim of the present retrospective study was to evaluate: (i) the longterm outcome of CNs treated with PEI; (ii) the differences between two different PEI techniques; (iii) the response of nodules according to their baseline liquid component.

\section{Subjects and methods}

\section{Study design}

Two institutions participated in the present study, the public Mauriziano Hospital of Turin and a Private Thyroid outpatient clinic in Foggia (Italy). Both institutions retrospectively reviewed all CNs consecutively treated with PEI. As inclusion criterion for the present study, nodules were followed up for at least 2 years after PEI. Follow-up data were collected for included cases. The study was submitted and approved by the local ethical committees of the two centers involved in the study. All participants gave their informed consent.

\section{Institutional guidelines in indicating thyroid PEI}

In the two thyroid centers, PEI was always performed by three operators (MZ, AM, MD) having more than 30 years of experience in ultrasound and ultrasound-guided techniques. The two institutions used the same selection criteria for nodule and patient for indicating PEI. As selection criteria for nodules a $\mathrm{CN}$ was: (i) with cystic component $>30 \%$ of its volume; (ii) persisting at 3-6 months after first drainage; (iii) cytologically classified as cystic (TIR1C) or not-neoplastic (TIR2) lesions according to the Italian classification system $(17,18)$ described in Table 1; (iv) without calcifications or fibrosis; (v) welldefined; (vi) without substernal extension. As selection criteria for patients, a subject was eligible for PEI if he had: (i) compressive symptoms or aesthetic complaints specifically correlated to the $\mathrm{CN}$; (ii) age > 18 years; (iii) normal thyroid function; (iv) no major comorbidities. In addition, the following features excluded patients from PEI: (i) patient with history of neck irradiation or familial thyroid cancer; (ii) nodule with uncertain clinical, ultrasonographic, or cytological characteristics; (iii) patient unable to cooperate during ethanol injection. The CNs were divided according to their cystic composition: cystic (CYS) > 90\%, mainly cystic (M-CYS) 75-90\%, mixed (MIX) 50-75\%, solid-mixed (S-MIX) $35-50 \%$ (19).

\section{PEI procedure}

The positioning of patient and operator were similar to those adopted during the FNA procedure, with the patient lying supine with neck in hyperextension. After skin sterilization, and under ultrasound guidance (US-guide), a 19-21 Gauge $(0.8-1 \mathrm{~mm})$ needle mounted on a $20 \mathrm{~mL}$ syringe were used to empty the contents of the cyst. Subsequently, 99\% ethanol was injected according to the two different protocols described in the following. The patient was instructed to indicate any feeling of pain. The needle tip was constantly monitored during the procedure in order to ensure that it remained within the cyst. Patients were offered further PEI sessions only when the cyst regrew with reappearance of compressive symptoms or aesthetic complaints, the initial cytology was TIR2 (i.e. benign), and the patient refused surgery. The protocols of treatment were different in the two centers and are described in Table 2.

Table 1 Categories of the 2010 and 2014 version of the Italian system for reporting of the thyroid FNA cytology.

\begin{tabular}{ll}
\hline 2010 version (17) & 2014 version (18) \\
\cline { 1 - 2 } TIR1: not adequate & $\begin{array}{l}\text { TIR1: not adequate } \\
\text { TIR1C: cystic }\end{array}$ \\
TIR2: benign/not neoplastic & TIR2: benign/not neoplastic \\
TIR3: indeterminate & TIR3A: low-risk indeterminate \\
& TIR3B: high-risk indeterminate \\
TIR4: suspicious for malignancy & TIR4: suspicious for malignancy \\
TIR5: malignant & TIR5: malignant \\
\hline
\end{tabular}


Table 2 PEI protocol of the two Institutions participating the study.

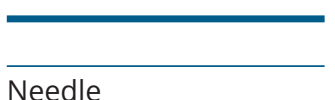

Needle

Treatment description

Session interval

\section{Post-PEI follow-up}

At both institutions participating to the study a clinical and ultrasound evaluation was performed at 1, 3, 6, and 12 months after the PEI. Successively, an annual re-evaluation was performed. At each evaluation, thyroid palpation, evaluation of symptom score (SYS) and ultrasonography were performed. Additional PEI sessions were offered to those patients with persistence of symptoms until their disappearance. No pre-established limits were set on the number of PEI session. Patients with no adequate response to repeated PEI were sent to surgery.

\section{Reference standard}

The percentage of cystic component of the nodule was calculated by the following formula: baseline volume $(\mathrm{mL})$ - final volume/baseline volume \%. The technique efficacy, expressed as volume reduction rate (VRR) of the nodule was defined as follows: nodule volume $(\mathrm{mL})$ after PEI/nodule volume (mL) before PEI x 100 (19). Nodules with a reappearance of cystic component larger than 0.5

\section{Foggia Outpatient Center}

PEI was performed with a 18 Gauge needle.

The entire procedure was monitored under US-guide and the patient asked to avoid talking and swallowing during the operative phases.

After the first drainage of about $90 \%$ of the liquid content of the nodule, immediately after sterile $95 \%$ ethanol was injected keeping the needle inside the cyst cavity, with a ratio of $0.3-0.5 \mathrm{~mL}$ ethanol $/ \mathrm{mL}$ of drained liquid. After a few seconds to let the hyperechoic intracystic cloud to disappear, the procedure was concluded by flushing the needle with lidocaine while withdrawing it. The ethanol left inside the nodule was not re-extracted and patients were discharged after a 30-min observation period.

The amount of ethanol injected for each session could be reduced in case of individual intolerance (i.e. injection on larger nodules was better tolerated).
A new treatment session was scheduled every four-five weeks up to the complete disappearance of liquid content of the nodule.

$\mathrm{mL}$ (e.g. $10 \times 10 \times 10 \mathrm{~mm}$ ) after PEI were defined as nonresponders and then re-treated with further PEI session.

The following symptoms were considered: pressure symptoms in the neck; difficulty in swallowing (dysphagia) and/or aesthetic complaint. An arbitrary three-score scale ( 0 =absent; 1 =moderate; $2=$ severe) was attributed to each symptom, as elsewhere described (7) and their sum indicated the severity (SYS) (range 0-6).

\section{Statistical analysis}

Continuous variables were expressed as mean \pm s.D. or median (interquartile range) and categorical variables as percentages. Student's t-tests were used for comparison between continuous variables and Pearson's correlation test for correlation analyses. A $P$ value $<0.05$ was considered statistically significant and correlations were considered significant for Pearson's correlation coefficient if $r \geq 0.25$. For comparison of categorical variables, the chi-square test or Fisher's exact test were used when appropriate. Data analyses were carried out using the Statistical Package for Social Sciences, Version 15.0 (SPSS@). 


\section{Results}

\section{Demographic data}

According to the previous selection criteria, a number of 358 nodules were enrolled for the present study, 152 from Turin and 206 from Foggia (Table 3). At baseline, patients from Turin were older than those from Foggia, and the amount of alcohol used in Turin was significantly higher. No difference between the two institutions was recorded in sex, median nodules' volume and number of PEI sessions.

\section{Clinical results}

SYS was significantly reduced after 6 months $(2.1 \pm 0.3$ vs $0.2 \pm 0.5, P<0.01)$ and at the end of the follow-up period $(0.2 \pm 0.4)$.

\section{Overall results of $\mathrm{PEI}$}

The 10-year follow-up data is shown in Table 4. Overall, 1-year VRR was significantly higher than that at 6 months $(P=0.0005)$. No other statistical difference was observed later. A significant difference of VRR between the two institutions was observed only at early visit (6 months) being VRR $79 \pm 19$ in Turin patients and $86 \pm 13$ in Foggia center $(P=0.002)$. The percentage of non-responders recorded in Turin and Foggia was 7 and 1.5\%, respectively. The recurrence rate was significantly lower in Turin than Foggia ( $14 \%$ vs $24 \%, P=0.001)$.

\section{Complications}

No major complications were reported. Transient pain was recorded in 4 (3\%) and 44 (21\%) in Turin and Foggia, respectively $(P<0.0001)$. Other minor side-effects were

Table 3 Main baseline features of the study series.

\begin{tabular}{|c|c|c|c|}
\hline & Turin & Foggia & $\boldsymbol{P}$ \\
\hline Patients $(n)$ & 152 & 206 & - \\
\hline Female (\%) & 70 & 72 & 0.59 \\
\hline Age (years) & $53 \pm 13$ & $45 \pm 14$ & $<0.001$ \\
\hline $\begin{array}{l}\text { Baseline nodules' } \\
\text { volume }(\mathrm{mL})\end{array}$ & $23 \pm 22$ & $24 \pm 27$ & 0.79 \\
\hline Cystic component & & & 0.17 \\
\hline$>90 \%$ (CYS) & $42(28 \%)$ & $70(34 \%)$ & \\
\hline 75-90\% (M-CYS) & $50(33 \%)$ & $61(30 \%)$ & \\
\hline $50-75 \%(\mathrm{MIX})$ & $51(33 \%)$ & $54(26 \%)$ & \\
\hline >35-50\% (S-MIX) & $9(6 \%)$ & $21(10 \%)$ & \\
\hline PEI sessions $(n)$ & $2 \pm 1$ & $2 \pm 1$ & 0.58 \\
\hline Ethanol injected (mL) & $15 \pm 13$ & $6 \pm 8$ & $<0.001$ \\
\hline
\end{tabular}

Table 4 Long-term VRR of the 358 cystic nodules. Data are presented as median (25-75 percentiles).

\begin{tabular}{lcc}
\hline Time points & Values \\
\cline { 1 - 1 } months & $84.53(72.88-93.58)$ \\
year & & $89.28(72.88-96.36)$ \\
5 years & $89.54(70.42-97.45)$ \\
10 years & $90.78(66.76-97.78)$ \\
\hline
\end{tabular}

Data are presented as median (25-75 percentiles).

reported with no difference between Turin and Foggia series: needles obstruction in $15(10 \%)$ and 13 (6\%) cases; hematoma in 0 and 2 (1\%) cases; fever in 0 and $6(3 \%)$ cases, respectively.

\section{Outcome of nodules based on their cystic composition}

A number of 192 nodules with a 10-year follow-up were considered for this analysis. The VRR of four subgroups, according to the baseline cystic component, is shown in Fig. 1. The VRR observed in CYS was significantly higher than that of M-CYS $(P<0.0001)$. The VRR of the latter was significantly higher than MIX $(P<0.0001)$. The VRRs of MIX and S-MIX were not statistically different $(P=0.08)$.

\section{Outcome of nodules undergone surgery}

In total, 13 non-responders (9 (7\%) in Turin series, and 4 (1.5\%) in Foggia series) underwent lobectomy (9 cases) or total thyroidectomy (4 cases). Among these cases, at histologic examination, a benign lesion was observed in 11 patients while one papillary cancer and one follicular variant of papillary cancer were found in the remaining two. Both cancers were nodules larger than $50 \mathrm{~mL}$ with bloody cystic content and a cytologic report of TIR1C. No complications after surgery were recorded in these cases.

\section{Discussion}

To the best of our knowledge, this is the only large multicenter study with long-term results of CNs treated with PEI. Our study showed a high efficacy of PEI in CNs with rapid shrinkage of nodule, stable effect at long-term follow-up, and low rate of non-responders. Some clinical issues can be fully discussed.

First, our data demonstrated that PEI was a valuable treatment for symptomatic thyroid CNs and results are largely better than what is generally considered as a 


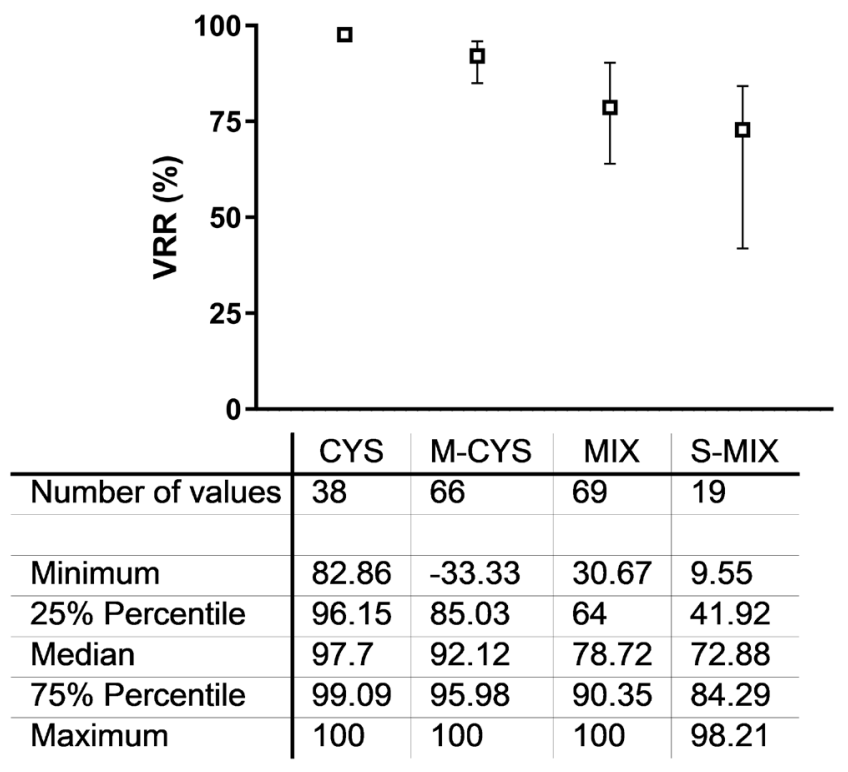

\section{Figure 1}

CYS, CN with cystic component in >90\%; M-CYS, CN with cystic component in 75-90\%; MIX, CN with cystic component in 50-75\%; S-MIX, CN with cystic component in $>35-50 \%$. The squares represent the median VRRs and the interquartile ranges.

satisfying outcome (20). Importantly, the overall VRR recorded early (i.e. 6-12 months after PEI) remained unchanged at 10-year follow-up and PEI was effective in $96 \%$ of patients. As a direct consequence, a significant reduction of symptoms was observed and the percentage of complications was very low.

Second, the results were not significantly influenced by the PEI procedure used; as a matter of fact, only at 6 months, we could observe a difference in percentage of non-responders between the two institutions, being this result not confirmed later. These data should corroborate the results recorded by Kim et al. (21) who described in 2005 the simple ethanol instillation after aspiration of fluid from CNs through the same needle. This should mean that any PEI procedure should be effective with good clinical outcome and low risks for patients. As the Turin intensive protocol showed a lower rate of recurrences (14\%) in comparison to Foggia group (24\%), this finding could support the indication to apply the single-shot approach at first, and the intensive protocol for the recurrent cases.

Third, as a further novelty, the PEI reliability was strongly associated with the cystic content of the nodule at baseline. Specifically, the larger the cystic component, the better the response to PEI. Nevertheless, an optimal result could be achieved also in medium-sized S-MIX nodules where the VRR (72\%) was higher than the baseline estimated liquid content (35-50\%); this result can be explained by a possible underestimation of liquid content at US and by the coagulative effect of alcohol on the solid part of nodule surrounding the cystic collections.

Fourth, the 14 patients (4\%) who failed to respond to PEI had a nodule larger than $50 \mathrm{~mL}$, they were referred for surgical removal and two papillary carcinomas were found on histology. Our data suggest applying PEI in CNs with volume between 4 and 30-35 mL to avoid an unsuccessful treatment, and addressing to surgery those with regrowth. The possibility that a malignant nodule is present after a not-diagnostic or benign cytology (17) should be taken into account. Therefore, PEI should be given only when the risk of cancer is as negligible as possible. In this regard, the following issues should be considered: (i) here we treated with PEI only CNs larger than $4 \mathrm{~mL}$ because it was demonstrated that the larger the volume of CNs the lower the risk of cancer (13); (ii) we performed PEI only after a double benign/cystic content cytologic report; even if biopsy has lower sensitivity in CNs, two unsuspicious cytologic samples can minimize the risk of false-negative; (iii) we performed PEI only when ultrasound features suggestive for cancer were absent $(22,23,24)$; 4) we addressed to surgery those cases with regrowth and tow cancers were found among 13 recurrent CNs.

Our multicentric study could finally contribute to give solid scientific evidence to consider PEI as the firstline treatment for CNs in future recommendations for the management of cytologically benign nodules.

Some strengths and limitations of the present study should be discussed. The strengths were the following: (i) the multicenter design with one public Hospital and one private clinic sharing the study; (ii) the large sample size enrolled; (iii) the unchanged procedure at each institution. As the major limitations, we may consider that the procedures were performed by highly experienced operators and this could influence positively the result in terms of efficacy. A prospective study with less experienced operators should be useful to confirm the herein recorded 1-year VRR.

In conclusion, the PEI was proven to be capable to contribute, alongside the thermal therapies (i.e. radiofrequency, laser, microwaves, etc.), for changing the management of patients with benign thyroid nodules. According to the aims of the present large multicenter study, we can report that: (i) the long-term outcome of CNs treated by PEI was excellent; (ii) this result was not 
influenced by the specific PEI technique used; (iii) the larger the cystic amount the higher the VRR. According to these data, predominantly or purely thyroid CNs that become symptomatic should first undergo US-guided percutaneous aspiration for both diagnostic and therapeutic purposes, and later they can be treated with PEI in case of relapse. This approach is based on the essential role of cytologic examination to rule out malignancy and the need of skilled PEI operators to achieve satisfactory clinical results.

\section{Declaration of interest}

The authors declare that there is no conflict of interest that could be perceived as prejudicing the impartiality of this study.

\section{Funding}

This research did not receive any specific grant from funding agencies in the public, commercial, or not-for-profit sectors.

\section{Author contribution statement}

M Deandra, A Mormile, R Garberoglio, surgeon and author of the paper; M Alberto, G Roberto, M Zingrillo: surgeons; ; P Trimboli, A Creanza, F Garino, S Bertolino, P P Limone: aexpert sonographers and visiting clinicians.

\section{References}

1 Burman KD \& Wartofsky L. Clinical practice. Thyroid nodules. New England Journal of Medicine 2015373 2347-2356. (https://doi. org/10.1056/NEJMcp1415786)

2 Bennedbaek FN \& Hegedüs L. Treatment of recurrent thyroid cysts with ethanol: a randomized double-blind controlled trial. Journal of Clinical Endocrinology and Metabolism 200388 5773-5777. (https:// doi.org/10.1210/jc.2003-031000)

3 Monzani F, Lippi F, Goletti O, Del Guerra P, Caraccio N, Lippolis PV, Baschieri L \& Pinchera A. Percutaneous aspiration and ethanol sclerotherapy for thyroid cysts. Journal of Clinical Endocrinology and Metabolism 199478 800-802. (https://doi.org/10.1210/ jcem.78.3.8126160)

4 Cho YS, Lee HK, Ahn IM, Lim SM, Kim DH, Choi CG \& Suh DC. 327 sonographically guided ethanol sclerotherapy for benign thyroid cysts: results in 32822 patients. American Journal of Roentgenology 2000174 213-216. (https://doi.org/10.2214/ajr.174.1.1740213)

5 Guglielmi R, Pacella CM, Bianchini A, Bizzarri G, Rinaldi R, Graziano FM, Petrucci L, Toscano V, Palma E, Poggi M et al. Percutaneous ethanol 331 injection treatment in benign thyroid lesions: role and efficacy. Thyroid 200414 125-332.

6 Valcavi R \& Frasoldati A. Ultrasound-guided percutaneous ethanol injection therapy in thyroid cystic nodules. Endocrine Practice 2004 10 269-275. (https://doi.org/10.4158/EP.10.3.269)

7 Zingrillo M, Torlontano M, Ghiggi MR, D'Aloiso L, Nirchio V, Bisceglia M \& Liuzzi A. Percutaneous ethanol injection of larger thyroid cystic nodules. Thyroid 19966 403-408. (https://doi. org/10.1089/thy.1996.6.403)

8 Gharib H, Hegedüs L, Pacella CM, Baek JH \& Papini E. Clinical review: nonsurgical, image-guided, minimally invasive therapy for thyroid nodules. Journal of Clinical Endocrinology and Metabolism 2013 98 3949-3957. (https://doi.org/10.1210/jc.2013-1806)
9 Reverter JL, Alonso N, Avila M, Lucas A, Mauricio D \& PuigDomingo M. Evaluation of efficacy, safety, pain perception and health-related quality of life of percutaneous ethanol injection as first-line treatment in symptomatic thyroid cysts. BMC Endocrine Disorders 201515 73. (https://doi.org/10.1186/s12902-015-0069-3)

10 Negro R, Colosimo E \& Greco G. Outcome, pain perception, and health-related quality of life in patients submitted to percutaneous ethanol injection for simple thyroid cysts. Journal of Thyroid Research 20172017 9536479. (https://doi. org/10.1155/2017/9536479)

11 Haugen BR, Alexander EK, Bible KC, Doherty G, Mandel SJ, Nikiforov YE, Pacini F, Randolph G, Sawka A, Schlumberger M et al. American Thyroid Association management guidelines for adult patients with thyroid nodules and differentiated thyroid cancer. Thyroid 201526 1-13.

12 Gharib H, Papini E, Paschke R, Duick DS, Valcavi R, Hegedüs L, Vitti P \& AACE/AME/ETA Task Force on Thyroid Nodules. American Association of Clinical Endocrinologists, Associazione Medici Endocrinologi, and European Thyroid Association medical guidelines for clinical practice for the diagnosis and management of thyroid nodules. Endocrine Practice 201016 (Supplement 1) 1-43. (https:// doi.org/10.4158/10024.GL)

13 Zingrillo M, Torlontano M, Chiarella R, Ghiggi MR, Nirchio V, Bisceglia M \& Trischitta V. Percutaneous ethanol injection may be a definitive treatment for symptomatic thyroid cystic nodules not treatable by surgery: five-year follow-up study. Thyroid 19999 763-767. (https://doi.org/10.1089/thy.1999.9.763)

14 Del Prete S, Caraglia M, Russo D, Vitale G, Giuberti G, Marra M, D'Alessandro AM, Lupoli G, Addeo R, Facchini G et al. Percutaneous ethanol injection efficacy in the treatment of large symptomatic thyroid cystic nodules: ten-year follow-up of a large series. Thyroid 200212 815-821. (https://doi.org/10.1089/105072502760339398)

15 Barile A, Quarchioni S, Bruno F, Ierardi AM, Arrigoni F, Giordano AV, Carducci S, Varrassi M, Carrafiello G, Caranci F et al. Interventional radiology of the thyroid gland: critical review and state of the art. Gland Surgery 20187 132-146. (https://doi.org/10.21037/ gs.2017.11.17)

16 Park HS, Yim Y, Baek JH, Choi YJ, Shong YK \& Lee JH. Ethanol ablation as a treatment strategy for benign cystic thyroid nodules: a comparison of the ethanol retention and aspiration techniques. Ultrasonography 201938 166-171. (https://doi.org/10.14366/ usg.18033)

17 Fadda G, Basolo F, Bondi A, Bussolati G, Crescenzi A, Nappi O, Nardi F, Papotti M, Taddei G, Palombini L et al. Cytological classification of thyroid nodules. Proposal of the SIAPEC-IAP Italian Consensus Working Group. Pathologica 2010102 405-408.

18 Nardi F, Basolo F, Crescenzi A, Fadda G, Frasoldati A, Orlandi F, Palombini L, Papini E, Zini M, Pontecorvi A et al. Italian consensus for the classification and reporting of thyroid cytology. Journal of Endocrinological Investigation 201437 593-599. (https://doi. org/10.1007/s40618-014-0062-0)

19 Mauri G, Pacella CM, Papini E, Solbiati L, Goldberg SN, Ahmed M \& Sconfienza LM. Image-guided thyroid ablation: proposal for standardization of terminology and reporting criteria. Thyroid 2019 29 611-618. (https://doi.org/10.1089/thy.2018.0604)

20 Papini E, Pacella CM \& Hegedus L. Diagnosis of endocrine disease: thyroid ultrasound (US) and US-assisted procedures: from the shadows into an array of applications. European Journal of Endocrinology 2014170 R133-R146. (https://doi.org/10.1530/EJE-130917)

21 Kim DW, Rho MH, Kim HJ, Kwon JS, Sung YS \& Lee SW. Percutaneous ethanol Injection for benign cystic thyroid nodules: is aspiration of ethanol-mixed fluid advantageous? American Journal of Neuroradiology 200526 2122-2127.

22 De los Santos ET, Keyani-Rofagha S, Cunningham JJ \& Mazzaferri EL. Cystic thyroid nodules: the dilemma of malignant lesions. Archives 
of Internal Medicine 1990150 1422-1427. (https://doi.org/10.1001/ archinte.150.7.1422)

23 Solbiati L, Livraghi T, Ballarati E, Ierace T \& Crespi L. Thyroid gland. In Ultrasound of Superficial Structures, pp. 50-85. Eds L Solbiati \& G Rizzatto. Edinburg: Churchill-Livingstone, 1995.
24 Zingrillo M, Collura D, Ghiggi MR, Nirchio V \& Trischitta V. Treatment of large cold benign nodules not eligible to surgery with percutaneous ethanol injection. Journal of Clinical Endocrinology and Metabolism 199883 3905-3907. (https://doi.org/10.1210/ jcem.83.11.5257)

Received 10 March 2020

Revised version received 6 July 2020

Accepted 7 August 2020 